Published in final edited form as:

Methods Mol Biol. 2019 ; 1960: 191-205. doi:10.1007/978-1-4939-9167-9_17.

\title{
Influenza-mediated Lung Infection Models
}

\author{
Charles E. McGee ${ }^{1}$, Christopher J. Sample ${ }^{1}$, Brita Kilburg-Basnyat ${ }^{2}$, Kristin Gabor ${ }^{3}$, \\ Michael B. Fessler ${ }^{3}$, Kymberly M. Gowdy2," \\ ${ }^{1}$ Duke Human Vaccine Institute, Duke University Medical Center, Durham, NC, 27710 \\ ${ }^{2}$ Department of Pharmacology and Toxicology, East Carolina University, Greenville, NC, 27858 \\ ${ }^{3}$ Immunity, Inflammation, and Disease Laboratory, National Institute of Environmental Health \\ Sciences, NIH, Research Triangle Park, NC, 27709
}

\section{Summary}

\begin{abstract}
Laboratory rodent influenza infection models have been and continue to be a critical tool for understanding virus-host interactions during infection. The incidence of seasonal influenza infections coupled with the need for novel therapeutics and universal vaccines highlight the need to uncover novel mechanisms of pathogenesis and protection. Mouse models are extremely useful for the evaluation of influenza vaccines and provide an invaluable tool to probe the immune response. This chapter describes the technique of intranasal inoculation of male C57BL/6J mice with an H1N1 strain of influenza (A/Puerto Rico/8/1934) and methods for assessing the optimum dose for infection, viral titers in lung tissue, and severity of disease.
\end{abstract}

\section{Keywords}

Intranasal inoculation; Influenza; H1N1; Body weight loss; Foci forming units; Lung damage

\section{Introduction}

Influenza infection is a significant cause of morbidity and mortality worldwide; the World Health Organization estimates that there are 3-5 million severe influenza cases every year, causing 250,000-500,000 deaths globally [1-3]. Influenza virus infections, both A and B, are seasonal and even though efficacious vaccines are available, factors such as viral genetic drift and mismatched vaccine strain selections can negatively impact the effectiveness of the vaccine and available anti-viral therapeutics. Additionally, individuals with known risk factors (elderly, obesity, hypertension, asthma, and heart disease) are at higher risk for severe disease including influenza viral pneumonia [4-6]. Therefore, laboratory studies focused on improving our understanding of the pathogenesis, immunobiology, and genetic drift of influenza viruses are critical for the development of next generation/universal vaccines [7].

\footnotetext{
*Corresponding Author: Department of Pharmacology and Toxicology, Brody School of Medicine, East Carolina University, 600 Moye Blvd, Room 6S-10, Greenville, NC 27858, Fax: 252-744-3203, Gowdyk14@ecu.edu.

25. It is recommended to develop a pathology grading scale to determine the extent and intensity of influenza induced pathology to correlate with other endpoints. If not working with a board-certified pathologist, representative lung pathology grading schemes can be found in the literature. However, the most accurate grading of lung pathology will be done by a blinded, board-certified pathologist.
} 
The murine influenza infection model has been fundamental to understanding the virus life cycle and replication as well as the host defense response. The utility of this laboratory model has led to better understanding of the viral replication kinetics, spread, and tropism as well as insight into the immune responses required to clear the virus. Additionally, rodent models of populations with known risk factors have allowed us to better understand the differing infection and immune response that may increase morbidity and mortality associated with an influenza infection [8]. However, many mechanisms of host-virus interactions remain unclear and further studies are needed to develop better vaccines and therapeutics.

Murine models of influenza infection have both advantages and disadvantages. Using mice provides a well characterized animal model with multiple options for differing genetics and phenotypes to understand vaccination response and characterize the immune response to influenza infection. Mouse breeding and reproduction is relatively quick and produces large litters thereby facilitating laboratory influenza studies of sufficient group sizes to achieve high statistical power. However, it should be acknowledged that there are differences in the mouse lung physiology and anatomy that may affect the extrapolation of data to human influenza infection. For instance, mice are obligate nose breathers whereas humans breathe utilizing the nose and the mouth [9]. Additionally, the branching of airways in the mouse is more simplistic than in the human lung, making the inhalation of the virus and its subsequent deposition different [10,11]. Influenza infection in mice requires a mouse adapted strain of influenza given that mice do not normally acquire and spread seasonal influenza strains [12]. Lastly, the morbidity of influenza lung infection in mice presents as weight loss and thus does not precisely model the respiratory syndrome seen in humans [12]. These differences between murine and human influenza infection should be considered when attempting to extrapolate data from laboratory infection studies.

Most mouse strains used for laboratory influenza infections are inbred thus creating numerous distinct models each of which is comprised of genetically identical individuals. However, it has been reported that the genetic background of the mouse can influence the susceptibility and immune response to influenza [13]. For instance, the A/J and DBA/J strains are more susceptible to influenza-induced pathology where as C57Bl/6 and BALB/c mice are considered more resistant to influenza induced pathology but exhibit significant weight loss post-infection and are therefore routinely used for lethal challenge research studies [13]. The variation in susceptibility amongst inbred strains of mice has been studied across multiple subtypes of influenza (H1N1, H3N2, avian and swine strains). It is thought that the variation in susceptibility is a result of genetic mutations in Mx1, Stat1, Pkr, Ifnar1, and/or Ncr1 that have been shown to influence the anti-viral response [14-18]. Additionally, wild caught mice are resistant to influenza infection based partially on $M x$ - or $M x+$ allele frequency [19-22]. Therefore, most strains of influenza used in mouse models require "mouse adaptation" to create a virus that will reproducibly infect and replicate in the murine respiratory tract and induce a weight loss phenotype.

Despite the differences in murine and human influenza infections, this model of viral infections is important and necessary to study and identify novel mechanisms of virus/host interactions and evaluate the protective efficacy of antiviral therapeutics and experimental 
vaccine formulations. This chapter will describe methods for studying a mouse-adapted influenza virus infection in mice. We have selected the infection of C57BL/6J male mice, a commonly used commercially available inbred strain, with an H1N1 strain of influenza (A/ Puerto Rico/8/1934; PR8), as an example. However, it should be noted that these methodologies can be used for the study of other influenza viruses capable of infecting various strains of mice.

\section{Materials}

\subsection{Infection}

1. Sterile, pharmaceutical grade, endotoxin-free, physiologic (0.9\%) saline or $1 \mathrm{X}$ phosphate-buffered saline (PBS)

2. Isoflurane

3. Isoflurane vaporizer

4. Isoflurane induction chamber (see Note 1)

5. Sterile $200 \mu \mathrm{L}$ pipet tips

6. Single channel $200 \mu \mathrm{L}$ pipette

7. Sterile $1000 \mu \mathrm{L}$ pipet tips

8. Single channel $1000 \mu \mathrm{L}$ pipette

\subsection{Monitoring weight loss after infection}

1. Scale accurate in the range of 10 to $50 \mathrm{~g}$ with at least a $0.01 \mathrm{~g}$ resolution.

2. Washable/autoclavable and shatter resistant flat bottom container (e.g., plastic $1000 \mathrm{~mL}$ beaker).

3. Mode of identification of individual experimental animals. Examples are tail markings or tattoos, ear tags, ear punches and injectable transponders (see Note 2).

\subsection{Quantification of lung viral titers using influenza virus focus forming assay (FFA)}

1. Sterile capped tubes (assorted)

2. Tube racks

\footnotetext{
1. The size of the isoflurane induction chamber should be sufficient to contain the animal with a normal posture but small enough to reduce "dead space." Additionally, ensure that the ports/tubing leading to the chamber are open to make sure the anesthetic vapor reaches the chamber. Isoflurane vaporizer and induction chamber should be used in combination with a carbon filter and/or Class II BSC or to minimize exposure of the user.

2. Mice should be tracked individually since weight loss/infectivity can be variable. The most affordable method is either tail marking using a "permanent" marker (requires periodic remarking every 2-3 days) or ear punching. Ear punching does not require anesthesia in rodents when performed by a skilled individual. Ear punching can generally be performed at approximately $15-17$ days of age, after the ear has "thinned." The ear punch procedure should be performed using clean gloves and a sanitized ear-punch. Manually restrain the animal and place the punch device on the pinna of the ear (external ear) in a location where you want to mark the animal for identification. Press firmly to punch with a commercially available ear punch tool to create a circular hole or semicircular notch through the ear. Bleeding after ear punching is uncommon and the animal can be released directly into the cage. Injectable transponders are implanted subcutaneously.
} 
3. Sterile pipet tips and serological pipets (assorted)

4. Pipetting devices

5. Tissue Homogenizer

6. Sterile 12-well flat-bottom tissue-culture-treated plate

7. Class II biological safety cabinet

8. $37^{\circ} \mathrm{C}$ cell culture incubator with $5 \% \mathrm{CO}_{2}$

9. $\quad 34-35^{\circ} \mathrm{C}$ cell culture incubator with $5 \% \mathrm{CO}_{2}$ (see Note 3 )

10. $37^{\circ} \mathrm{C}$ water bath (see Note 4 )

11. Centrifuge

12. Vortex

13. Timer

14. Inverted microscope

15. White light box

16. Hemocytometer or other cell counting device

17. Freezers, -20 and $-80^{\circ} \mathrm{C}$

18. Refrigerator, $4^{\circ} \mathrm{C}$

19. Liquid nitrogen storage

20. Madin-Darby canine kidney (MDCK) cells (see Note 5)

21. MDCK cell culture maintenance medium: Minimum Essential Medium supplemented with Earl's salts and L-glutamine (MEM), $10 \%$ fetal bovine serum (FBS; non-heat-inactivated), $100 \mathrm{U} / \mathrm{mL}$ penicillin- $100 \mu \mathrm{g} / \mathrm{mL}$ streptomycin, 1 $\mathrm{mM}$ sodium pyruvate, and $1 \mathrm{X}$ non-essential amino acids

22. $\quad 0.05 \%$ Trypsin-EDTA

23. Sterile PBS (1X) for cell culture

24. $1 \mathrm{mg} / \mathrm{mL}$ TPCK-trypsin (TPCK: L -(tosylamido-2-phenyl) ethyl chloromethyl ketone) in $1 \mathrm{mM} \mathrm{HCl}(\mathrm{pH} 3), 20 \mathrm{mM} \mathrm{CaCl}_{2}$

25. Virus diluent: Dulbecco's Modified Eagle Medium (DMEM), $100 \mathrm{U} / \mathrm{mL}$ penicillin- $100 \mu \mathrm{g} / \mathrm{mL}$ streptomycin, $1 \%$ bovine serum albumin (BSA; Fraction V), and $25 \mathrm{mM}$ HEPES; supplemented with $1.0 \mu \mathrm{g} / \mathrm{mL}$ TPCK-trypsin immediately prior to use (see Note 6)

\footnotetext{
3. Influenza viruses such as PR8 often grow optimally at a slightly cooler temperature $\left(34-35^{\circ} \mathrm{C}\right)$ than the temperature recommended for MDCK cell growth $\left(37^{\circ} \mathrm{C}\right)$.

4. Media, PBS and trypsin should be pre-warmed to $37^{\circ} \mathrm{C}$ before putting on MDCK cells to avoid cold shocking the cells.

5. There are several sub-types of MDCK cells. MDCK (NBL-2) (ATCC® CCL-34) cells are recommended for use in the FFA procedure described here.

6. If fungal contamination is anticipated/possible supplement with $0.25 \mu \mathrm{g} / \mathrm{mL}$ Amphotericin B.
} 
26. FFA overlay: DMEM, $100 \mathrm{U} / \mathrm{mL}$ penicillin- $100 \mu \mathrm{g} / \mathrm{mL}$ streptomycin, $0.1 \%$ BSA (Fraction V), $3.7 \mathrm{~g} / \mathrm{L}$ sodium bicarbonate, $0.01 \%$ w/v DEAE-dextran, and 1.2\% Avicel® RC-591 NF microcrystalline cellulose (MCC) w/v; supplemented with $1.0 \mu \mathrm{g} / \mathrm{mL}$ TPCK-trypsin immediately prior to use (see Note 6 )

27. $10 \%$ neutral buffered formalin (NBF)

28. Wash buffer: PBS $1 \mathrm{X}$ with $0.1 \%$ Tween ${ }^{\circledR} 20$

29. Permeabilization buffer: $1 \mathrm{X}$ PBS with $10 \mathrm{mM}$ glycine and $0.5 \%$ Triton $^{\mathrm{TM}} \mathrm{X}-100$

30. Avidin stock solution: $10 \mathrm{mg} / \mathrm{mL}$ in $1 \mathrm{X}$ PBS and $5 \mathrm{mM}$ sodium azide

31. Biotin stock solution: $10 \mathrm{mg} / \mathrm{mL}$ in DMSO

32. Antibody diluent: $1 \mathrm{X}$ PBS, $1 \%$ BSA (Fraction V), $0.1 \%$ Tween ${ }^{\circledR} 20$, and $1 \%$ $(w / v)$ non-fat dry milk powder

33. Anti-influenza A virus NP antibody clones A1 and A3; biotin conjugated and pre-mixed 1:1

34. Streptavidin conjugated to horseradish peroxidase (HRP)

35. Immunohistochemical (IHC) staining reagent for peroxidase-labeled conjugate detection (see Note 7)

\subsection{Identification of Lung Pathology after Influenza Infection}

1. Surgical tools (e.g., scissors, scalpel)

2. $3 \mathrm{~mL}$ Syringe

3. Thin catheter or a 19 gauge blunt needle, able to fit in mouse trachea

4. Surgical sutures, dental floss, or small string

5. $10 \% \mathrm{NBF}$ solution

6. Paraffin

7. Device for sectioning tissues to $5 \mu \mathrm{m}$ thickness such as a cryostat.

8. Positively charged glass slides

9. Hematoxylin and eosin stains

\section{Methods}

\subsection{Preparation of Virus Inoculum}

1. Thaw a stock ampule of virus on ice. Virus stock should be thawed just before use for optimal infectivity. The use of repeatedly freeze/thawed virus aliquots is not recommended.

\footnotetext{
7.KPL TrueBlue ${ }^{\mathrm{TM}}$ Substrate (Sera Care, Milford, MA) is recommend however, other vendors may have comparable products.
} 
2. Dilute virus sample with sterile saline or PBS (usually two or three dilutions may be necessary differing by 10 -fold in the range of $1: 100$ to 1:50,000) (see Note 8) to make the dose of virus per mouse about a $50 \mu \mathrm{L}$ volume (See Note 9).

3. Virus and diluted virus should be kept on ice or at $4^{\circ} \mathrm{C}$ throughout preparation and before administration. Concentration of virus given to mice will depend on study and preparation of stock virus (see Note 10).

\subsection{Intranasal Inoculation of Virus}

1. Fill the isoflurane vaporizer with isoflurane to fill line (do not fill while vaporizer is on; do not over fill the vaporizer).

2. Turn on oxygen tank maintaining 0.5-1 liter/minute flow of oxygen.

3. Place the mouse in the induction chamber

4. Turn on isoflurane vaporizer at 4-5\% (See Note 11).

5. Watch mouse until it is deeply anaesthetized (confirmed by the absence of reflexes on the footpad) (see Note 12).

6. Mix virus inoculum well by pipetting up and down

7. The mouse is held by its scruff and the inoculum is gradually released into the nostrils (see Note 13) with the help of a pipette. This method of dosing is shown in Figure 1. The rate of release should be gradual to allow the mouse to inhale the inoculum without forming bubbles. When performed correctly a rapid increase in the breathing rate should be observed.

8. After dosing, the mouse should be placed back in its cage on its back to ensure no bedding blocks nasal passages and to prevent residual virus inoculum from exiting the front of the nostrils. For prolonged anesthesia the cage should have a mild heat source to prevent hypothermia, care must be taken not to over-heat the animals.

9. Visually monitor the mouse until it is moving normally about the cage.

\footnotetext{
8. Pipetting small volumes can increase error. Making serial dilutions to achieve the desired dose/concentration of virus increases experiment-to-experiment reproducibility.

9.40-50 $\mu \mathrm{L}$ is the recommended inoculum dose. Smaller volumes may become stuck in the nasal cavity and expelled or may not evenly distribute in the lung and thus increase variability.

10. There are multiple sources that may cause lab-to-lab variation in PR8 mouse infection models. The virus is available from multiple commercial sources and stocks can be generated via growth in different culture systems (chicken eggs, MDCK cells, etc). The level of expertise of the laboratory, the source/strain of the mice, age of the mice, propagation method for the virus, etc. may all contribute to variation. Therefore, it is recommended that an MLD (minimal dose that is lethal to a certain percentage (e.g., 50\%) of animals) is performed prior to the initiation of experimental studies to identify the specific dosages to be used that fits the experimental goals. Figure 4 shows an example of a survival study performed to identify an MLD50 dose of PR8 in C57BL/6J male mice.

11. The use of a calibrated vaporizer provides the most consistent and safe delivery of inhalant anesthesia. If a standard vaporizer is not available, isoflurane may be suspended in glycerol/paraffin/mineral oil and administered on cotton or gauze in an induction chamber and/or nose cone. Use of isoflurane without a precision vaporizer has a very small margin of safety. Animals can easily become overdosed if not constantly monitored.

12. If animals are not anesthetized to a sufficient level and wake up too quickly they may expel the inoculum instead of breathing it in fully. This can create variation in infection rates.

13. The technician should try to evenly distribute the inoculum between the two nostrils.
} 


\subsection{Evaluation of Weight Loss after Infection}

1. Monitor weight loss and recovery throughout the duration of the model to assess morbidity associated with infection. Due to the daily flux in mouse weights, define a 2-hour window each day when the mice will be weighed. To evaluate weight, zero or tare the scale with the container in place before use (see Note 14). Gently pick up the mouse by the base of the tail and transfer the animal to the container on the scale. Once a steady reading can be made, which is generally when the mouse has stopped moving around, record the weight. Ensure that a baseline weight measurement has been taken immediately prior to infection (see Note 15) for each mouse. This initial weight is necessary for the calculation of the daily percent weight loss.

2. Calculate the daily percent weight change for each animal over a 14 day study duration. Determine the difference between the pre- and post-infection weights and divide this difference by the pre-infection weight to obtain percent body weight change. Alternatively, divide post-infection body weight by baseline (preinfection) body weight for each mouse to obtain the percent of original body weight. Example weight loss curves over time of mice given differing doses of PR8 are shown in Figure 2.

\subsection{Quantification of Viral Titer in Lung Tissue by FFA}

1. Excise the lung tissue into a pre-weighed, labeled tube.

2. Re-weigh the tube and subtract the empty tube weight to calculate the weight of the lung tissue.

3. Homogenize the lung tissue in $1-2 \mathrm{ml}$ of virus diluent and store on ice or $4^{\circ} \mathrm{C}$ (see Notes 16-17).

4. The day before assay, trypsinize the MDCK cells, count and dilute cell number to desired seeding density (see Note 18) with MDCK cell culture maintenance medium. Add $1.0 \mathrm{~mL}$ of diluted cells to each well of a 12-well assay plate. Incubate overnight (16-24 hours) at $37^{\circ} \mathrm{C}, 5 \% \mathrm{CO}_{2}$.

5. Prepare a set of six tubes for each lung sample in which to perform 10-fold serial dilutions by adding $450 \mu \mathrm{L}$ of virus diluent per tube.

6. Perform 10-fold serial dilutions for each sample. Briefly, add $50 \mu \mathrm{L}$ of homogenized lung to the first tube and pulse vortex. Repeat this process by

\footnotetext{
14. The scale with weight container in place should be checked periodically to ensure a zero reading when empty, re-zero as necessary.

15 . Because of daily flux in weight the baseline weight can be calculated as the average of multiple measurements taken up to $2-3$ days prior to infection.

16. FFA does not need to be performed immediately after necropsy. Samples can be batched up, frozen and stored at $-80^{\circ} \mathrm{C}$ for later analysis. Snap freezing tissues using liquid nitrogen is recommended.

17. If lung tissue is not homogenized completely, pieces of connective tissue can clog pipet tips resulting in inaccurate pipetting and/or cause MDCK cytotoxicity thereby negatively impacting FFA performance and reproducibility. Therefore, clarifying lung homogenates by centrifugation $(\geq 10,000 \times \mathrm{g})$ at $4^{\circ} \mathrm{C}$ for 5 minutes is recommended.

18. An MDCK cell monolayer confluence of $75-85 \%$ is recommended, to achieve this cell density seed cells at $1.66 \times 10^{5}$ cells $/ \mathrm{mL}-$ $2.08 \times 10^{5}$ cells/mL between 16 and 24 hours prior to the assay respectively. In house evaluation of seeding density to achieve desired confluence may be necessary.
} 
serially transferring $50 \mu \mathrm{L}$ of $10^{-1}$ to $10^{-2} ; 10^{-2}$ to $10^{-3}$; etc. to create the remaining dilutions making sure to change pipet tips and vortex after each dilution.

7. Remove culture medium from the assay plate wells and wash the MDCK monolayers once with 1X PBS (do not allow cells to dry).

8. Remove the PBS wash and add $100 \mu \mathrm{L}$ of lung homogenate serial dilutions to the plate. Each dilution should be plated in duplicate wells. One 12-well plate allows for the analysis of 6 serial 10-fold dilutions of a single sample plated in duplicate.

9. Incubate the assay plates at $34-35^{\circ} \mathrm{C}, 5 \% \mathrm{CO}_{2}$. for 1 hour. Rock assay plates every 10-15 minutes to ensure even distribution of inoculum and to prevent drying of the cell monolayer.

10. Add $1 \mathrm{~mL}$ of $1.2 \%$ Avicel MCC-based FFA overlay [23] per well. It is not necessary to remove inoculum prior to addition of overlay unless inoculum cytotoxicity is anticipated.

11. Incubate the assay plates at $34-35^{\circ} \mathrm{C}, 5 \% \mathrm{CO}_{2}$. for 2 days (see Note 19).

12. Fix plates by adding $1 \mathrm{~mL}$ of $10 \%$ NBF directly to the overlay of each well and incubating for 30-60 minutes at room temperature (see Note 20).

13. Remove FFA overlay/NBF and wash plates $3 X$ with wash buffer $(0.5 \mathrm{~mL}$ per well per wash) (see Note 21).

14. Remove wash buffer, add $0.5 \mathrm{~mL}$ permeabilization buffer per well and incubate 15-20 minutes at room temperature.

15. Remove permeabilization buffer and wash plates $3 \mathrm{X}$ with wash buffer $(0.5 \mathrm{~mL}$ per well per wash).

16. Dilute avidin stock solution $1: 10,000$ in $1 \mathrm{X}$ PBS. Remove wash buffer from plate, add $200 \mu \mathrm{L}$ diluted avidin blocking solution per well and incubate 15 minutes at room temperature with periodic rocking.

17. Remove avidin blocking solution and wash plates $3 \mathrm{X}$ with wash buffer $(0.5 \mathrm{~mL}$ per well per wash).

18. Dilute biotin blocking stock solution $1: 1,000$ in $1 X$ PBS. Remove wash buffer from plate, add $200 \mu \mathrm{L}$ diluted biotin blocking solution per well and incubate 15 minutes at room temperature with periodic rocking.

\footnotetext{
19. Assay incubation times will vary from 2-3 days depending on influenza virus strain-specific growth kinetics in MDCK cells. Whenever a new virus is being evaluated some optimization of incubation time may be required.

20. Plates may also be fixed with $-20^{\circ} \mathrm{C} 80 \%$ acetone in $1 \mathrm{X}$ PBS. If using this methodology; remove overlay, wash monolayers twice with $1 \mathrm{X}$ PBS, add $2.0 \mathrm{~mL}$ pre-chilled acetone fixative per well, incubate for 10-12 minutes at room temperature, remove acetone, allow plates to briefly dry (1-5 minutes; excess drying can increase background), wash three times, and proceed to avidin solution incubation. Two step fixation/permeabilization using NBF and Triton-X generally results in improved IHC staining.

21. Plates may be stored up to one-week post-fixation at $4^{\circ} \mathrm{C}$ in $1 \mathrm{X}$ PBS with $1 \% \mathrm{BSA}$ and $0.1 \%$ Tween 20 provided sufficient buffer is used to prevent plates from drying out during storage.
} 
19. Remove biotin solution and wash plates $3 \mathrm{X}$ with wash buffer $(0.5 \mathrm{~mL}$ per well per wash).

20. Dilute biotin labeled anti-influenza A virus NP clones A1 and A3 1:1,000 (or tested optimal concentration) in antibody diluent. Remove wash buffer from plate, add $200 \mu \mathrm{L}$ diluted A1 and A3 solution per well and incubate 1 hour at room temperature with periodic rocking every 10-15 minutes (see Note 22).

21. Remove $\mathrm{A} 1$ and $\mathrm{A} 3$ solution and wash plates $3 \mathrm{X}$ with wash buffer $(0.5 \mathrm{~mL}$ per well per wash).

22. Dilute streptavidin-HRP 1:1,000 (or tested optimal concentration) in antibody diluent. Remove wash buffer from plate, add $200 \mu \mathrm{L}$ diluted streptavidin-HRP solution per well and incubate 30 minutes at room temperature with periodic rocking every $10-15$ minutes.

23. Remove streptavidin-HRP solution and wash plates $5 \mathrm{X}$ with wash buffer $(0.5 \mathrm{~mL}$ per well per wash).

24. Develop plate using IHC staining reagent for peroxidase-labeled conjugate detection in accordance with manufacturer protocols (see Note 23).

25. Quench developing reaction in accordance with staining reagent manufacturer protocol.

26. Dry plates in the dark overnight or according to staining reagent manufacturer's protocol and manually enumerate foci using a white light box for increased contrast. The titer for a given dilution series should be scored using the well with the highest number of distinct and countable foci. The foci forming units (FFU) titer for a given sample is calculated as follow:

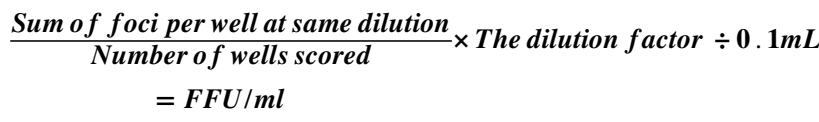

For lung homogenates the calculated FFU/mL and measured tissue weight should be used to express titers as FFU/g tissue weight to normalize for differences in the size/weights of lung samples assayed.

\subsection{Lung Histology}

1. Euthanize mouse on selected day after infection based on experimental design/ endpoints. Figure 3 show examples of lung histology on days 3 and 7 after infection, respectively (see Notes 24).

\footnotetext{
22. When titering samples of mouse origin, such as lung homogenates, it is necessary to use biotin-tagged anti-influenza antibodies and to sequentially incubate plates with avidin and biotin blocking solutions to decrease assay background. However, this protocol can be adapted for the analysis of influenza samples of non-mouse origin. If titering non-mouse samples, avidin and biotin blocking is not necessary and anti-influenza A virus NP clones A1 and A3 that are not biotin labeled should be used in conjunction with an antimouse HRP conjugated secondary antibody.

23. For KPL TrueBlue ${ }^{\mathrm{TM}}$ Substrate (SeraCare Life Sciences) is recommended use $200 \mu \mathrm{L}$ per well and quench with tap water once staining becomes sufficiently intense but before background begins to develop.
} 
2. Surgically expose and isolate the trachea taking care to keep it intact. Taking care not to puncture or cut the lungs, open the chest cavity to expose the lungs.

3. Gently inflate the lungs with $10 \%$ NBF. This technique can be done via pushing the fluid through a syringe and an attached thin catheter inserted in a small hole in the trachea. Tie off the trachea with surgical sutures to ensure the liquid does not leave lungs. Monitor inflation of lungs as to not overfill resulting in artifactual damage.

4. Surgically remove the whole lungs.

5. Immerse the infused tissue in $10 \% \mathrm{NBF}$ for a minimum of 48 hours.

6. Fix, paraffin embed, section (Figure 3 , tissues were sectioned at $5 \mu \mathrm{m}$ ), and mount the sections on positively charged glass slides.

7. Stain with hematoxylin and eosin (H\&E).

8. Evaluate lung histology.

9. Correlate the histologic evaluations with weight loss (see Note 24).

\section{Acknowledgements}

The Virology Unit of the Duke Regional Biocontainment Laboratory (RBL) under the directorship of Dr. Gregory D. Sempowski, which received partial support for construction from the National Institutes of Health, National Institute of Allergy and Infectious Diseases (UC6-AI058607). This work was also supported by the National Institutes of Health, National Institute of Environmental Health Sciences (Z01 ES102005 to MBF).

\section{References}

1. Flannery B, Chung JR, Thaker SN, et al. (2017) Interim Estimates of 2016-17 Seasonal Influenza Vaccine Effectiveness - United States, February 2017. MMWR Morb Mortal Wkly Rep 66 (6):167_ 171. doi:10.15585/mmwr.mm6606a3 [PubMed: 28207689]

2. Dugan VG, Blanton L, Elal AIA, et al. (2017) Update: Influenza Activity - United States, October 1November 25, 2017. MMWR Morb Mortal Wkly Rep 66 (48):1318-1326. doi:10.15585/ mmwr.mm6648a2 [PubMed: 29216030]

3. Blanton L, Alabi N, Mustaquim D, et al. (2017) Update: Influenza Activity in the United States During the 2016-17 Season and Composition of the 2017-18 Influenza Vaccine. MMWR Morb Mortal Wkly Rep 66 (25):668-676. doi:10.15585/mmwr.mm6625a3 [PubMed: 28662019]

4. Eilers R, Krabbe PF, van Essen TG, et al. (2013) Assessment of vaccine candidates for persons aged 50 and older: a review. BMC Geriatr 13:32. doi:10.1186/1471-2318-13-32 [PubMed: 23586926]

5. Powell ND, Allen RG, Hufnagle AR, et al. (2011) Stressor-induced alterations of adaptive immunity to vaccination and viral pathogens. Immunol Allergy Clin North Am 31 (1):69-79. doi:10.1016/ j.iac.2010.09.002 [PubMed: 21094924]

6. Dutkowski R (2010) Oseltamivir in seasonal influenza: cumulative experience in low- and high-risk patients. J Antimicrob Chemother 65 Suppl 2:ii11-ii24. doi:10.1093/jac/dkq012 [PubMed: 20215131]

7. Erbelding EJ, Post D, Stemmy E, et al. (2018) A Universal Influenza Vaccine: The Strategic Plan for the National Institute of Allergy and Infectious Diseases. J Infect Dis doi:10.1093/infdis/jiy103

24. At day 3 post-infection there is minimal pathology with some inflammation/apoptosis of the epithelium of the larger airways and some immune cells present in tissue whereas at day 7 post-infection extensive perivascular and peribronchiolar infiltrates as well as alveolar congestion are observed. 
8. Thangavel RR, Bouvier NM (2014) Animal models for influenza virus pathogenesis, transmission, and immunology. J Immunol Methods 410:60-79. doi:10.1016/j.jim.2014.03.023 [PubMed: 24709389]

9. Trabalon M, Schaal B (2012) It takes a mouth to eat and a nose to breathe: abnormal oral respiration affects neonates' oral competence and systemic adaptation. Int J Pediatr 2012:10.1155/2012/207605

10. Winkler-Heil R, Hofmann W (2016) Modeling particle deposition in the Balb/c mouse respiratory tract. Inhal Toxicol 28 (4):180-191. doi:10.3109/08958378.2016.1148801 [PubMed: 26986953]

11. Hogan BL, Barkauskas CE, Chapman HA, et al. (2014) Repair and regeneration of the respiratory system: complexity, plasticity, and mechanisms of lung stem cell function. Cell Stem Cell 15 (2): 123-138. doi:10.1016/j.stem.2014.07.012 [PubMed: 25105578]

12. Bouvier NM, Lowen AC (2010) Animal Models for Influenza Virus Pathogenesis and Transmission. Viruses 2 (8):1530-1563. doi:10.3390/v20801530 [PubMed: 21442033]

13. Trammell RA, Toth LA (2008) Genetic susceptibility and resistance to influenza infection and disease in humans and mice. Expert Rev Mol Diagn 8 (4):515-529. doi:10.1586/14737159.8.4.515 [PubMed: 18598231]

14. Srivastava B, Blazejewska P, Hessmann M, et al. (2009) Host genetic background strongly influences the response to influenza a virus infections. PLoS One 4 (3):e4857. doi:10.1371/ journal.pone.0004857 [PubMed: 19293935]

15. Gazit R, Gruda R, Elboim M, et al. (2006) Lethal influenza infection in the absence of the natural killer cell receptor gene Ncr1. Nat Immunol 7 (5):517-523. doi:10.1038/ni1322 [PubMed: 16565719]

16. Bergmann M, Garcia-Sastre A, Carnero E, et al. (2000) Influenza virus NS1 protein counteracts PKR-mediated inhibition of replication. J Virol 74 (13):6203-6206 [PubMed: 10846107]

17. Haller O, Arnheiter H, Lindenmann J (1976) Genetically determined resistance to infection by hepatotropic influenza A virus in mice: effect of immunosuppression. Infect Immun 13 (3):844854 [PubMed: 178595]

18. Koerner I, Kochs G, Kalinke U, et al. (2007) Protective role of beta interferon in host defense against influenza A virus. J Virol 81 (4):2025-2030. doi:10.1128/JVI.01718-06 [PubMed: 17151098]

19. Staeheli P, Grob R, Meier E, et al. (1988) Influenza virus-susceptible mice carry Mx genes with a large deletion or a nonsense mutation. Mol Cell Biol 8 (10):4518-4523 [PubMed: 2903437]

20. Staeheli P, Haller O, Boll W, et al. (1986) Mx protein: constitutive expression in 3 T3 cells transformed with cloned Mx cDNA confers selective resistance to influenza virus. Cell 44 (1): 147-158 [PubMed: 3000619]

21. Vanlaere I, Vanderrijst A, Guenet JL, et al. (2008) Mx1 causes resistance against influenza A viruses in the Mus spretus-derived inbred mouse strain SPRET/Ei. Cytokine 42 (1):62-70. doi: 10.1016/j.cyto.2008.01.013 [PubMed: 18334301]

22. Haller O, Staeheli P, Kochs G (2007) Interferon-induced Mx proteins in antiviral host defense. Biochimie 89 (6-7):812-818. doi:10.1016/j.biochi.2007.04.015 [PubMed: 17570575]

23. Matrosovich M, Matrosovich T, Garten W, et al. (2006) New low-viscosity overlay medium for viral plaque assays. Virol J 3:63. doi:10.1186/1743-422X-3-63 [PubMed: 16945126] 

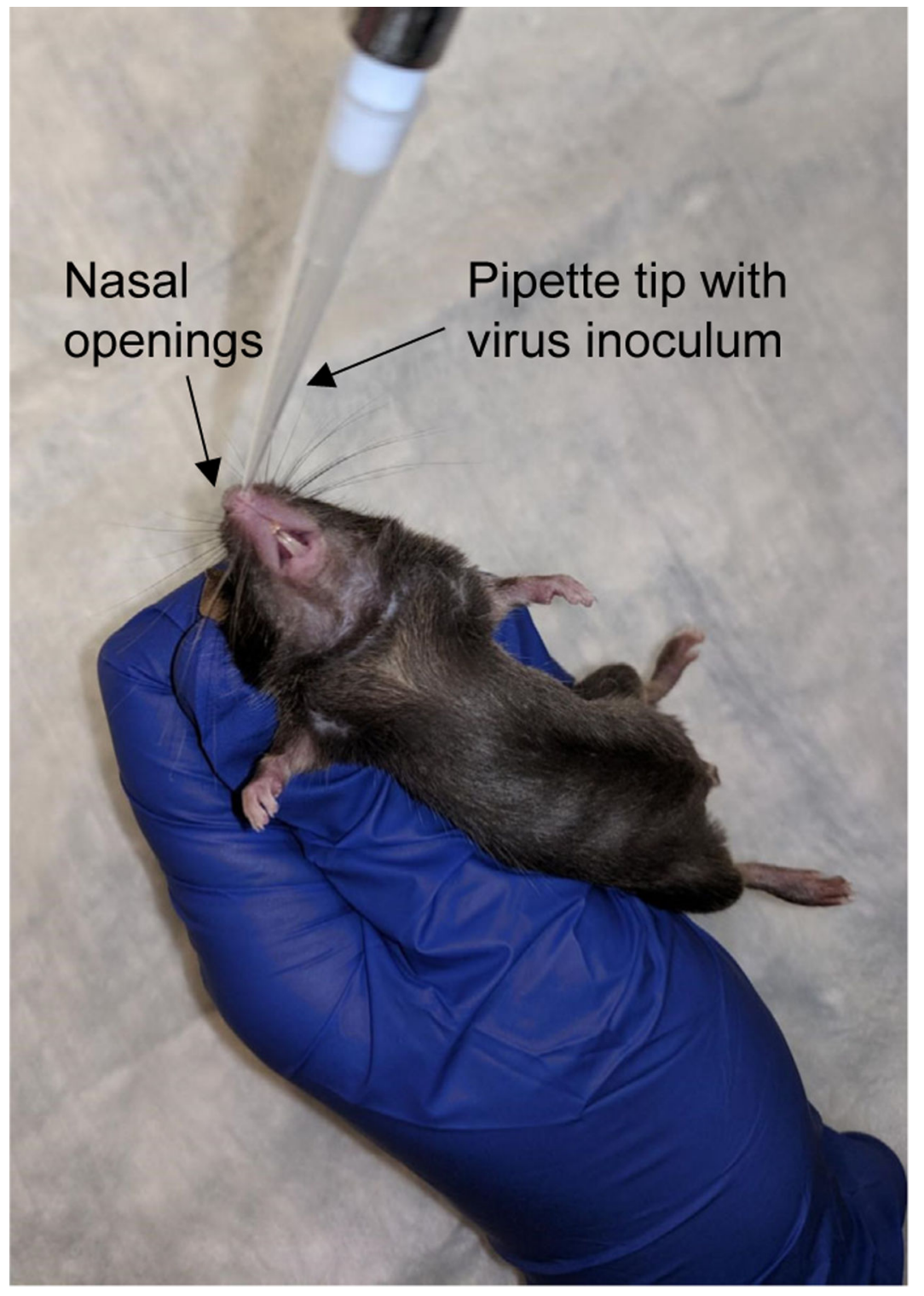

Figure 1.

Picture illustrating the intranasal inoculation of virus to an anesthetized mouse. 


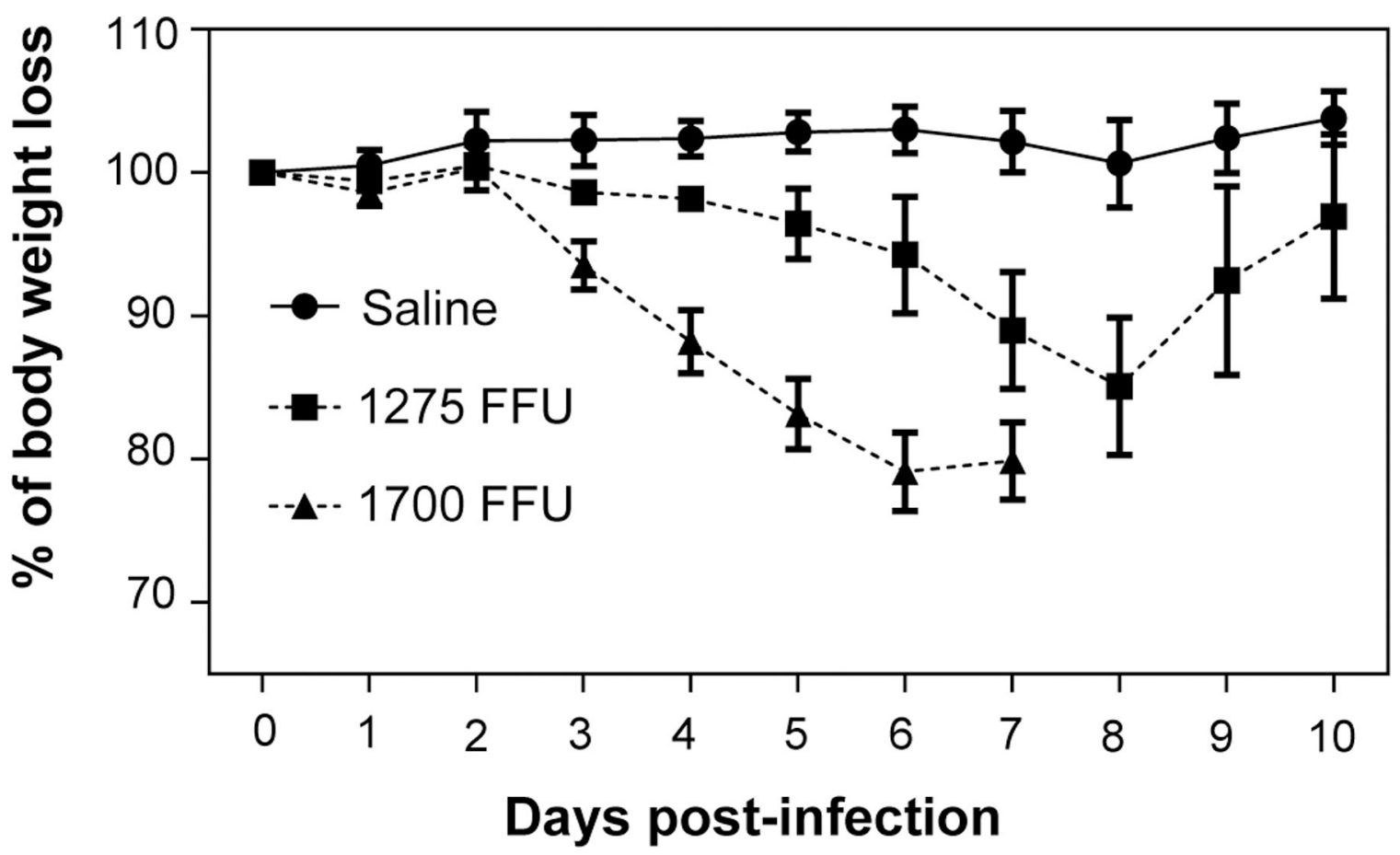

Figure 2.

Percentage of weight loss of male C57BL/6 mice after saline or PR8 infection (either 1275 or $1700 \mathrm{FFU})$. Mice were euthanized if they lost more than $20 \%$ of their original body weight. 

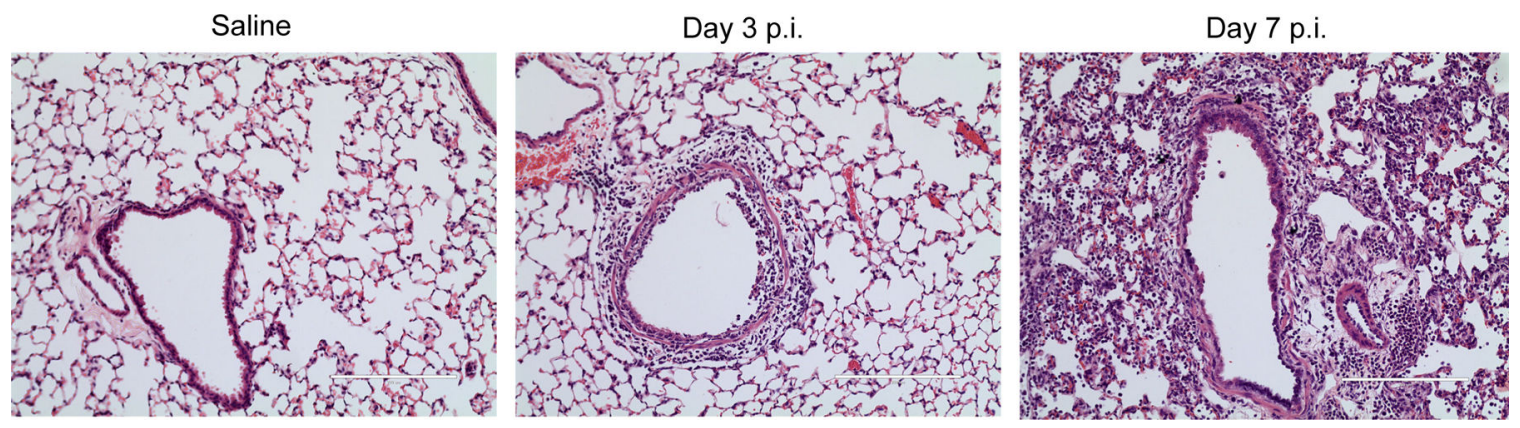

Figure 3.

Lung histology of C57BL/6 mouse after saline or PR8 infection (day 3 or 7 post infection (p.i.)). H\&E stain shown at $200 \times$ magnification. 

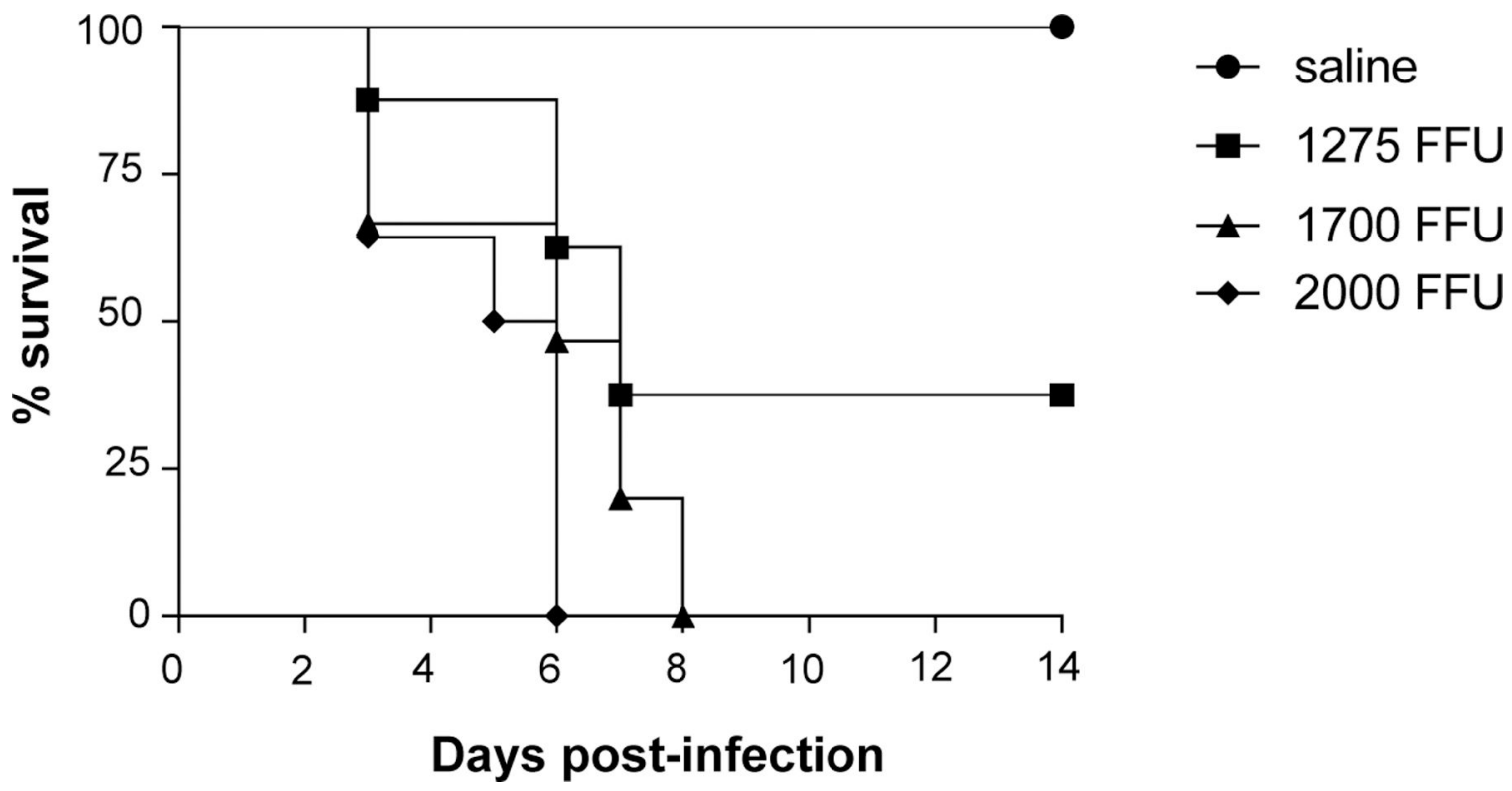

Figure 4.

Percentage of survival of male C57BL/6 mice after saline or PR8 infection (either 1275, 1700 , or 2000 FFU). Mice were euthanized if they lost more than $20 \%$ of their original body weight per institutional animal use guidelines. 